SUBJECT AREAS: BIOINSPIRED MATERIALS

WETTING

FLUIDS

FLUIDICS

Received

22 May 2013

Accepted

25 September 2013

Published

11 October 2013

\section{Correspondence and} requests for materials should be addressed to Y.Z. (zhengym@buaa. edu.cn)

* These authors contributed equally to this work.

\title{
Bioinspired tilt-angle fabricated structure gradient fibers: micro-drops fast transport in a long-distance
}

\author{
Yuan Chen ${ }^{1 *}$, Lin Wang ${ }^{1 *}$, Yan Xue' ${ }^{1}$ Lei Jiang ${ }^{1,2} \&$ Yongmei Zheng'
}

\begin{abstract}
'Key Laboratory of Bio-Inspired Smart Interfacial Science and Technology of Ministry of Education, School of Chemistry and Environment, Beihang University, Beijing, 100191 (P. R. China), ${ }^{2}$ Beijing National laboratory of Molecular Science, Institute of Chemistry, Chinese Academy of Science, Beijing, 100190 (P. R. China).
\end{abstract}

Issues of surfaces, e.g., inspired from beetle's back, spider silk, cactus stem, etc., become the active area of research on designing novel materials in need of human beings to acquire fresh water resource from air. However, the design of materials on surface structure is little achieved on controlling of micro-scale drop transport in a long distance. Here, we report the ability of micro-drop transport in a long distance on a bioinspired Fibers with Gradient Spindle-knots (BFGS), which are fabricated by tilt angle dip-coating method. The micro-drop of $\sim 0.25 \mu \mathrm{L}$ transports in distance of $\sim 5.00 \mathrm{~mm}$, with velocity of $0.10-0.22 \mathrm{~m} \mathrm{~s}^{-1}$ on BFGS. It is attributed to the multi-level cooperation of the release energy of drop coalescence along the gradient spindle-knots, in addition to capillary adhesion force and continuous difference of Laplace pressure, accordingly, water drops are driven to move fast directionally in a long distance on BFGS.

iological models of water collection are revealed on natural surfaces with unique excellent-designed microstructures $^{1-5}$. For example, desert beetle's back is utilized to collect water from humid air because of alternate hydrophobic and hydrophilic micro-regions ${ }^{1}$. A cacus O. Microdasys, which originates from the Chihuahua Desert, owning an integrated multifunctional system composed of well-distributed conical spines and trichomes on the cactus stem that facilitates efficient fog collection ${ }^{6}$. Besides a wonderful mechanical property ${ }^{7}$, the capture silk of spider has a water collecting ability resulted from a unique fiber structure that forms after the 'wet-rebuilt's characterized by periodic 'spindle-knots, joints' structure made of random nanofibrils and aligned nanofibrils respectively. Inspired by the creature structure features ${ }^{9-12}$, biomimetic replications of water harvesting systems open the new way to design fog collection materials ${ }^{13-15}$. In previous researches, water collection materials have been designed by the cooperation of surface energy gradient ${ }^{16-18}$ and Laplace pressure in difference ${ }^{8}$. Some technologies have been utilized such as dip-coating ${ }^{3-14}$, electrondynamics ${ }^{15}$, fluid-coating ${ }^{19}$, and micro-fluidic controlling techniques ${ }^{20}$. These excellent materials are expected to apply in high efficiency water collection device ${ }^{21,22}$, humidity sensors ${ }^{23,24}$, fluid-controlling, and micro-reactor devices ${ }^{25,26}$. However, the spindle-knot structures fabricated via previous coating method still limit to the water transport in distance, where the uniform as-distributed spindle-knots along fibers can't further drive the droplet to a long distance, e.g., the transport of micro-drop (e.g., $\sim 0.25 \mu \mathrm{L}$ ) in long distance (e.g., $\sim 5.00 \mathrm{~mm}$ ) along the structure gradient fiber surface, which hasn't been reported so far. Here, to obtain a kind of material with directional water drop transport ability, a bioinspired fiber with gradient spindle-knots (BFGS) is designed carefully, and fabricated by the tilted dip-coating method based on the Rayleigh instability break-up into gradient droplets ${ }^{27-29}$. It is found that the BFGS may realize the water transport in direction from smaller spindle-knots to bigger ones, which is not observed on the uniform fiber or uniform spindle-knot fiber. This finding opens a way to fabricate novel gradient functional fiber and offers the insight into the design of surface material to control the fluids movement.

\section{Results}

Fabrication and structure feature of bioinspired fibers. Inspired by water directional movement on wetted spider silk with spindle-knots, a new structure with gradient spindle-knots is designed to realize the fast directional movements of tiny drops in a long distance. Figure 1a shows the optical images on fabrication process of bioinspired fibers with gradient spindle-knots, via in-situ observation by using a high-speed CCD camera (Phantom v9.1, Vision Research, America). A uniform nylon fiber $(\sim 18 \mu \mathrm{m})$ is fixed on a support with $\theta$ angle to the horizontal line. The nylon fiber is firstly immersed into a polymer solution, Polyvinylidene Fluoride 
a

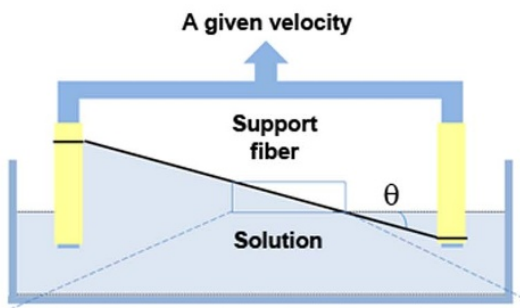

The process with gradient spindle-knots

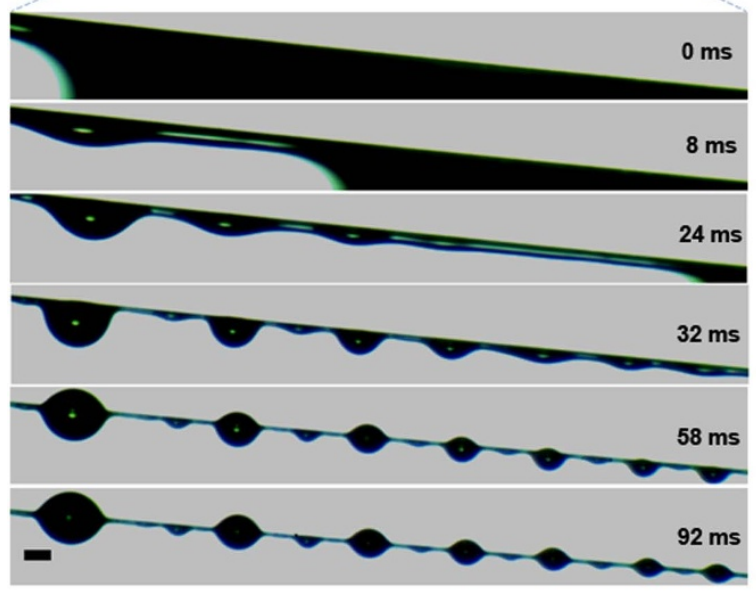

b

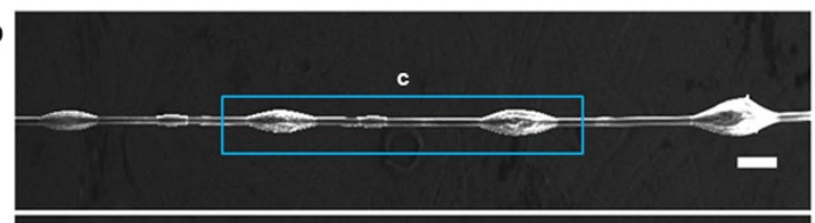

C

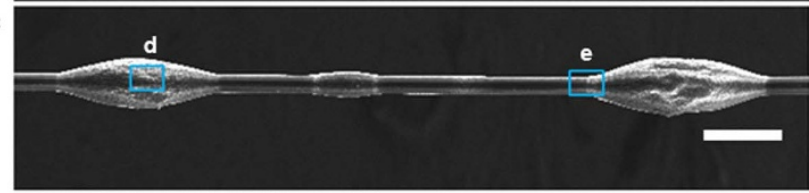

d

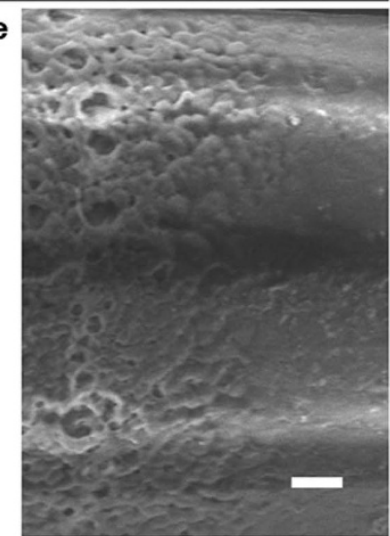

Figure $1 \mid$ Fabrication of BFGS and SEM images. (a), The scheme shows the whole fabrication process. A uniform nylon fiber $(\sim 18 \mu \mathrm{m})$ fixed on a support is soaked into a polymer solution (PVDF/DMF) with $\theta$ angle to the horizontal line and then drawn out at a given velocity. The optical images show that a cylindrical PVDF film breaks into gradient polymer droplets beginning from up to down. The first spindle-knot is bigger than next one and so on. All the spindle-knots are sequence gradient in heights. (b), SEM images of BFGS with gradient four spindle-knots. (c), Two spindle-knots in the middle. (d), There are nano-protrusions at the maximum diameter of spindle-knot. (e), The random nano-porous structure at the minimum diameter of the spindle-knot (joint). Scale bars, $200 \mu \mathrm{m}$ (a), $100 \mu \mathrm{m}$ (b,c), $1 \mu \mathrm{m}$ (d,e).

(PVDF, $\mathrm{M}_{\mathrm{w}}=530$ 000, Aldrich) dissolved in N,N-dimethylformamide (DMF), and then tilted-angle drawn out at a given velocity. As shown in Fig. 1a, initially, a slope PVDF film is covered on the fiber surface at the titled solid-liquid interface with gradient thickness (at $0 \mathrm{~ms}$ ). And then it gradually spontaneously breaks into polymer droplets attributed to the Rayleigh instability ${ }^{1,2}$ beginning from up to down (at $\sim 8-24 \mathrm{~ms}$ ). The break-up process of the slope film is accompanied by the film sliding down (at $\sim 8 \mathrm{~ms}$ ). The polymer droplets automatically form symmetrical oval due to surface tension to reach the lowest energy. After stable condition of polymer droplets (in $\sim 32-92 \mathrm{~ms}$ ), the BFGS would be formed excellently from gradient polymer droplets (at $\sim 92 \mathrm{~ms}$ ). There are two reasons to explain the results of gradient spindle-knots. Firstly, the first spindle-knot forming at the earliest break place utilizes the surrounding solution to form the biggest one, which will lead to reduce the size of the second one. At the same reason, the next one is less than the previous one (Fig. 1a). Secondly, the solution covering on the fiber will gradually decrease resulting from sliding down. Some solution comes back to the solution reservoir due to the film rapidly sliding. The thickness of film is greatly related to the result of polymer droplet break up, it is estimated that the heights of polymer droplets are ranged from $\sim 186$ to $\sim 51 \mu \mathrm{m}$ in the time of $\sim 92 \mathrm{~ms}$, and the widths of them are ranged from $\sim 289$ to $\sim 160 \mu \mathrm{m}$. When the solvent evaporate, the PVDF spindle-knots are formed along the fiber. As having been reported $^{14}$, when the fiber is drawn out horizontally, the sizes of these PVDF spindle-knots are usually in the uniform distribution. Interestingly, the fiber is drawn out at a tilted angle to the horizontal line, and the polymer film can be gradient thickness. Thus result of the Rayleigh instability break-up induces the sizes of these PVDF spindle-knots to decrease gradually, accordingly, BFGS forms effectively.

Microstructure on the surface of BFGS is observed by scanning electron microscopy (SEM, QuantaEFG250, FEI, America). Figure 1b-e show the SEM images of fiber (Fig. 1b) composed of four different sizes of spindle-knots from smaller to bigger. The height of spindleknots are $\sim 59 \mu \mathrm{m}, \sim 74 \mu \mathrm{m}, \sim 88 \mu \mathrm{m}$ and $\sim 118 \mu \mathrm{m}$, and the width of spindle-knots are $\sim 162 \mu \mathrm{m}, \sim 206 \mu \mathrm{m}, \sim 235 \mu \mathrm{m}$ and $\sim 268 \mu \mathrm{m}$, respectively. The four spindle-knots open the half of apex-angles of $\sim 25.0^{\circ}, \sim 28.0^{\circ}, \sim 28.5^{\circ}, \sim 31.0^{\circ}$ for geometry feature (smaller to bigger). Figure 1c shows the SEM images of two middle size spindle-knots as the representative. The right one is bigger than the left one. Figure $1 c-d$ shows the gradient of roughness on the surface of spindle-knot and joint. There are nano-protrusions at the maximum diameter of the spindle-knot (Fig. 1c) and random nano-porous structure at the minimum diameter of the spindle-knot (joint, Fig. 1d), which is mimicking the random and aligned nanofibrils on the spider silk $^{2}$. Every spindle-knot and every joint have the similar features of roughness gradients, but gradient sizes of continuous spindle-knots along fiber form distinct feature of BFGS.

To further reveal how the fabrication conditions affect the geometric parameters (i.e., height and width) and gradient extent, a series of gradient spindle-knots is fabricated carefully under different solution concentrations and tilted angles, which are shown by the optical images and size statistical data (see Fig. S1-S2). The concentration of the polymer solution is $7 \%, 8 \%, 9 \%$, or $10 \%$ (weight percentage of PVDF in DMF) and the title angle is $0^{\circ}, 5^{\circ}, 10^{\circ}$, or $15^{\circ}$ with the same draw-out velocity of $\sim 200 \mathrm{~mm} \mathrm{~min}^{-1}$. The fabricated fibers can be divided into three different types according to the shape of the gradient knots on them. In the green frame images, there are no obvious gradient structures on the spindle-knots. It results from too bigger title angle and too higher concentration. Due to a bigger angle, the polymer film fractures too fast to stay enough solution on the fiber. And higher polymer solution concentration increases the result because of the larger solution film gravitation. These two reasons will lead the solution film fracture quickly when the fibers just leave the solution surface. There is little solution on the fiber to break 
into obvious gradient spindle-knots or make the gradient spindleknots too short. In the images with red frame, the obvious gradient spindle-knots appear. The coating film thickness is affected by the solution concentration and title angle, and the film thickness further influence the formation of gradient spindle-knots. The effect of the concentration has been discussed by reported paper ${ }^{14}$. For coating technique to form solution film onto a fiber, the thickness of solution film is related to the concentration and viscosity of solution, drawout velocity and surface tension, which is regulated by capillary number (Ca) with $\mathrm{Ca}=\eta v / \gamma$, where $\eta$ is the solution viscosity; $v$ is the draw-out velocity; $\gamma$ is the solution surface tension. The polymer solution viscosities and surface tensions have been measured (see Supporting information, Tab. S1). The viscosities increase from $\sim 144$ to $\sim 460 \mathrm{mPa} \cdot \mathrm{s}$ for polymer solutions with different PVDF concentrations, while their surface tensions change from $\sim 36.38$ to $\sim 36.82 \mathrm{mN} \mathrm{m}^{-1}$. The angle to the horizontal line exists to make the solution film present a triangular shape when the fibers leave the solution from left to right. The gravitation of the solution film decreases from left to right on the fiber, and the length of the solid-liquid contact line is also reduced. So on the same fiber, the Ca varies with different positions, which lead to the thickness of film decreases from left to right on the fiber. The thickness determines the volume of the solution on the fiber. According to the length of two spindle-knots with $\mathrm{L}=\lambda d$, where $\lambda$ is the coefficient and $d$ is the diameter of fiber, the size of spindle-knots is determined by the volume of solution in L range on the fiber. So the height and width of spindle-knots are decreased from left to right. The lengths of pitches between two spindle-knots are also decreasing. As in our experiments, there are two effect factors for the formation of excellent gradient spindle-knots. If the angle is too bigger or the solution concentration is too higher, the solution film breaks too fast to slide down, which will lead to not enough PVDF solution on the fiber. So the gradient spindle-knots are not obvious or enough long to be used in water directional movement. Under $0^{\circ}$ angle to the horizontal line condition, there are periodic spindle-knots as the same as reported previously ${ }^{14}$. To further reveal how the size of the gradient spindleknots varies at optimized fabrication conditions, we select the gradient spindle-knots with solution concentrations of $7 \%, 8 \%, 9 \%$, and $10 \%$ in $5^{\circ}$ to the horizontal line. The statistical data about the height and width of the gradient spindle-knots and pitch between spindleknots are shown in Figure S2a-c, respectively. The data are obtained by five repeated experiments. We define as initial position (i.e., $0 \mathrm{~mm}$ ) of spindle-knots with $\sim 3 \mathrm{~mm}$ far from the fiber support. The chart shows that height and width of gradient spindle-knots on the same fiber are declined with a linear trend, which is determined by the thickness of solution film decreasing from left to right on the fiber. At high concentration (10\%), the slope rate is bigger than low concentration (7\%) with height and width. At 7\%, the height is from $\sim 0.09$ to $\sim 0.05 \mathrm{~mm}$, the width gradient from $\sim 0.35$ to $\sim 0.21 \mathrm{~mm}$, while the height and width ranges from $\sim 0.17$ to $\sim 0.07 \mathrm{~mm}$ and $\sim 0.50$ to $\sim 0.24 \mathrm{~mm}$ at $10 \%$, respectively. The distances of two spindle-knots have no obvious difference with the concentration for the relative position of every spindle-knot. The largest pitches are close to $1.5 \mathrm{~mm}$ in length. The average values for largest pitch of two spindle-knots in red frame are list in Table 2. But the higher title angle $\left(\sim 15^{\circ}\right)$ is not conducive to useful pitches in the gradient of fiber. These investigations allow us to evaluate the water directional movement ability of gradient spindle-knots fibers.

Water drop transport in one direction in a long distance. What is interesting is that BFGS we prepared display a property to transport water drops in a long distance. In our previous studies ${ }^{2,14,15,19,30,31}$, we reproduced the structural features similar to that of wetted spider silks. Specifically, the tiny water droplets directional driving on spindle-knots and a big drop pinning between two spindle-knots (uniform spindle-knots) were investigated significantly. However, directional movements of tiny water drops are usually limited in a short distance on surfaces of most previous as-designed fibers. Here, the water directional movement of the gradient spindle-knots can be carried out under a humidity of more than $90 \%$ via fog flow at rate of $\sim 30 \mathrm{~cm} \mathrm{~s}^{-1}$, which is observed by CCD camera. As shown in Figure 2, there are four spindle-knots from smaller to bigger one on fiber (the left top), with the width ranged from $\sim 180$ to $\sim 400 \mu \mathrm{m}$ and height from $\sim 75$ to $\sim 120 \mu \mathrm{m}$. The pitches are not more than $\sim 1.5 \mathrm{~mm}$ in length. The whole process is in the time of $\sim 130 \mathrm{~s}$. In details, at $\sim 84.0 \mathrm{~s}$, water drops $(1,2,3)$ are condensed on the spindleknots. In the time of $\sim 125.0 \mathrm{~s}$, the water drops grow and meantime the drops coalesce and move towards to bigger spindle-knots direction. Subsequently, drops 1, 2 and 3 coalesce into drop 4, which are moving to the right about $2 \mathrm{~mm}$ at $\sim 120 \mathrm{~s}$. And drop 5 and 6 coalesce into drop 7 . In the time of $\sim 130$ s, drop 4,7 and 8 coalesce into drop 9. All whole process, the water is directionally moving across four spindle-knots in distance of $\sim 5.0 \mathrm{~mm}$. Figure $2 \mathrm{~b}$ shows the details of water drop coalescence and movement in direction at $\sim 84-128.44 \mathrm{~s}$, where the drops $(1+2$ +3 ) fast coalesce into drop 4 at $\sim 84.40-84.50 \mathrm{~s}$; drops $(4+8)$ coalesce together at $\sim 128.36 \mathrm{~s}$; drops $(4+8+7)$ coalesce directionally into drop 9 at $\sim 128.44$ s, respectively. The BFGS can be continuous to perform water drop transport and display the repeatability as shown in Fig. S3. In contrast, no obvious movements of water drops in a long distance appear on a uniform nylon fiber with PVDF coating and uniform spindle-knots fiber when they are horizontally placed under a humidity of more than $90 \%$ via fog flow at rate of $\sim 30 \mathrm{~cm} \mathrm{~s}^{-1}$, where the water drops are coalesced with surrounding drops and stay on the original position (on the uniform nylon fiber, see Fig. S4a) or stably hang on two uniform spindle-knots (on the uniform spindle-knots fiber, see Fig. S4b). In our investigation, pitch of two spindle-knots influences the distance of water transport. If the pitches of two spindle-knots are more than $1.5 \mathrm{~mm}$ in length, droplets doesn't usually move to fardistance another larger spindle-knot, e.g., a pitch of $\sim 1.8 \mathrm{~mm}$, which water transport is not conducive to a long distance (see Fig. S5).

The process of water drops coalescence and transport can be different on BFGS with weak or strong gradient spindle-knots. Figure 3a shows the water drop behaviors of coalescence on weak gradient spindle-knots of BFGS. Three gradient spindle-knots (Fig. 3a, the top) in turn have $\sim 41 \mu \mathrm{m}, \sim 56 \mu \mathrm{m}$, and $\sim 58 \mu \mathrm{m}$ in height and $\sim 163 \mu \mathrm{m}, \sim 221 \mu \mathrm{m}$, and $\sim 244 \mu \mathrm{m}$ in width, respectively. The largest pitch is $\sim 0.9 \mathrm{~mm}$. The BFGS is placed horizontally, and a humidifier provides a humidity of $90 \%$. Five drops $(1,2,3,4,5)$ which have almost equal volumes including three ones (drops $1,3,5$ ) covering on single spindle-knot and two ones (drops 2,4) on joints (at $\sim 1.28 \mathrm{~s})$ coalesce into one bigger drop $(1+2+3+4+5)$ at $\sim 1.32-1.36 \mathrm{~s}$, hanging on between two bigger spindle-knots, in which process the bigger drop doesn't hang in the middle of BFGS. If the first drop (i.e., drop 1) is defined as the initial point (at $\sim 1.28$ s), the last bigger water drop (i.e., drop $(1+2+3+4+$ 5)) obtained by coalescence and movement (at $\sim 1.36 \mathrm{~s}$ ) is defined as the final point. The transport length $\mathrm{L}_{\mathrm{a}}$ of drop is $\sim 1.41 \mathrm{~mm}$ (see Fig. 3a, the distance between two vertical dash lines) in time of $\sim 1.36 \mathrm{~s}$. As for the strong gradient spindle-knots of fiber (Fig. 3b), where there are the five gradient spindle-knots (Fig. 3b, the top) with height of $\sim 35 \mu \mathrm{m}, \sim 41 \mu \mathrm{m}, \sim 56 \mu \mathrm{m}, \sim 58 \mu \mathrm{m}, \sim 81 \mu \mathrm{m}$ and width of $\sim 139 \mu \mathrm{m}, \sim 163 \mu \mathrm{m}, \sim 221 \mu \mathrm{m}, \sim 244 \mu \mathrm{m}, \sim 302 \mu \mathrm{m}$, in turn, respectively. The largest pitch is $\sim 1.1 \mathrm{~mm}$. Water drops grow on the BFGS at $\sim 2.04 \mathrm{~s}$. At $\sim 2.08 \mathrm{~s}$, the first four drops $(1,2,3,4)$ coalesce into a bigger one $(1+2+3+4)$ hanging on two spindle-knots (the third and forth spindle-knots), where the transport length of drop is $\sim 2.70 \mathrm{~mm}\left(\mathrm{~L}_{\mathrm{b} 1}\right)$ (see both vertical dash line, at $\sim 2.04-2.08 \mathrm{~s}$ ). This process is similar with Fig. $3 \mathrm{a}$, at the time $0-1.36 \mathrm{~s}$. The drop is coalesced with a small drop $((1+2+3+4), 5)$ on the right (at $\sim 2.08 \mathrm{~s})$ and the last drop $(1+2+3+4+5)$ moves to right point 


\section{a}
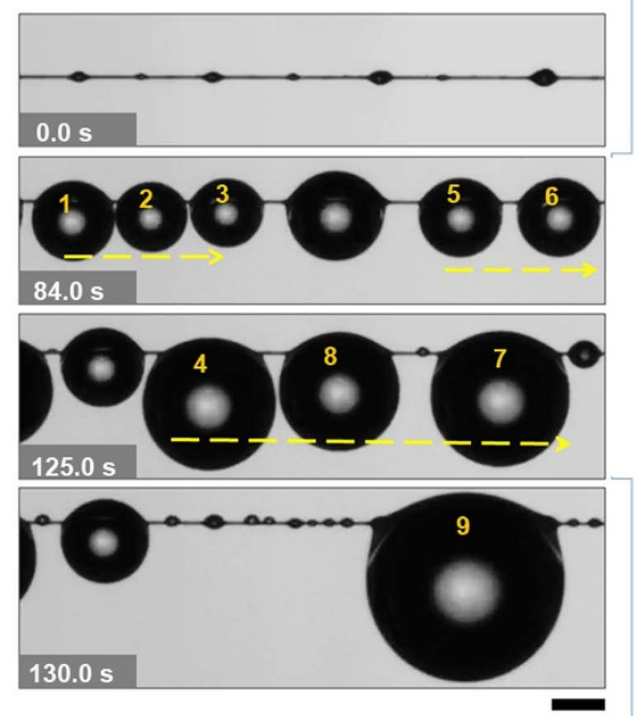

b
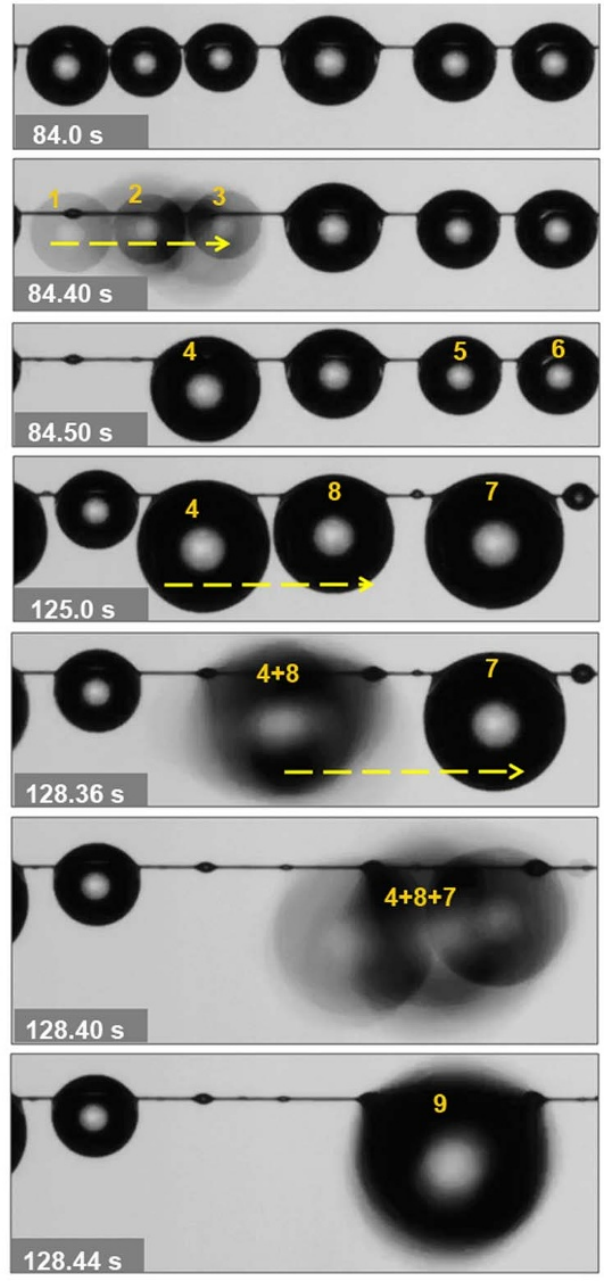

Figure 2 Water drops directional movement for a long distance on BFGS. (a), The gradient spindle-knots are from smaller to bigger. Water condensed drops $(1,2,3,5,6)$ form on the spindle-knots (at $\sim 84 \mathrm{~s}$ ) and they coalesce into bigger drops $(4,8,7)$, respectively, and they move fast towards the biggest spindle-knots with $\sim 5.0 \mathrm{~mm}$, to coalesce into a biggest drop 9 on the bigger spindle-knot at $\sim 130 \mathrm{~s}$. (b), The details of the water drops in directional movement. Some situations of dynamic coalescence and movement show the drops $(1+2+3 \rightarrow 4)$ at $\sim 84.40-84.50 \mathrm{~s}$; drops $(4+8)$ at $\sim 128.36 \mathrm{~s}$; drops $(4+8+7 \rightarrow 9)$ at $\sim 128.44 \mathrm{~s}$, respectively. Scale bars, $500 \mu \mathrm{m}$.

hanging on two biggest spindle-knots on the fiber (at $\sim 2.12 \mathrm{~s}$ ). The transport length $\left(\mathrm{L}_{\mathrm{b} 2}\right)$ of drop is $\sim 3.76 \mathrm{~mm}$ (see Fig. $3 \mathrm{~b}$, the distance between both vertical dash lines, at $\sim 2.12 \mathrm{~s}$ ). It indicates that a longer distance (i.e., $\sim 3.76 \mathrm{~mm}$ ) of drop movement appears along the strong gradient spindle-knots than that (i.e., $\sim 1.41 \mathrm{~mm}$ ) of weak gradient spindle-knots.

Furthermore, we select a gradient fiber with three different spindle-knots (i.e., height of $\sim 43 \mu \mathrm{m}, \sim 130 \mu \mathrm{m}, \sim 217 \mu \mathrm{m}$; width of $\sim 152 \mu \mathrm{m}, \sim 435 \mu \mathrm{m}$, and $\sim 565 \mu \mathrm{m}$, respectively; the largest pitch is $\sim 1.1 \mathrm{~mm}$ ). Water directional movement process is recorded by using CCD. In process of water drop movement as shown in Figure 4, it is found that the direction tendency of drop coalescence can also further be normal sequence and reverse sequence. In normal sequence (as shown in Fig. 4a), the observation is focused on behaviors of three water drops with volumes: $\Omega_{\mathrm{D} 1}<\Omega_{\mathrm{D} 2}<\Omega_{\mathrm{D} 3}$ ( $\sim 0.40 \mu \mathrm{L}, \sim 0.63 \mu \mathrm{L}, \sim 1.24 \mu \mathrm{L}$, respectively) (see Fig. $4 \mathrm{a}$ ). The process of water drops coalescence is in manner of $(\mathrm{D} 1+\mathrm{D} 2)+$ D3. In details, the drop D1 coalesces with drop D2 at $\sim 1.4 \mathrm{~ms}$. Drop $\mathrm{D}(1+2)$ moves towards bigger spindle-knots and is hanging on the middle spindle-knot via the second mode (at $\sim 9.8 \mathrm{~ms}$ ). With the water drops growing, drop $\mathrm{D}(1+2)$ is merging with drop D3 at $\sim 11.2 \mathrm{~ms}$. Due to the biggest spindle-knot with the most capillary force, the drop $\mathrm{D}(1+2)+3$ is hanging stably on the biggest one at $\sim 26.8 \mathrm{~ms}$. The length of movement $\left(\mathrm{L}_{\mathrm{a}}\right)$ is $\sim 2.8 \mathrm{~mm}$ at the whole coalescence process. In reverse sequence of droplet coalescence, the observation is focused on behaviors of three water drops with volumes: $\Omega_{\mathrm{D} 1^{\prime}} \approx \Omega_{\mathrm{D} 2^{\prime}} \approx \Omega_{\mathrm{D} 3^{\prime}}(\approx 0.67 \mu \mathrm{L})$ (see Fig. $\left.4 \mathrm{~b}\right)$. The process of water drop coalescence is in manner of $\left(\mathrm{D} 2^{\prime}+\mathrm{D} 3^{\prime}\right)+\mathrm{D} 1^{\prime}$. In details, at $\sim 2.80 \mathrm{~ms}$, the drop $\mathrm{D} 2^{\prime}$ and $\mathrm{D} 3^{\prime}$ firstly combine together. The drop $\mathrm{D}\left(2^{\prime}+3^{\prime}\right)$ finally hangs stably on the biggest spindle-knots at $\sim 8.4 \mathrm{~ms}$. With the drops growing, the drop $\mathrm{D} 1^{\prime}$ moves towards to the right due to the first way at $\sim 960.4 \mathrm{~ms}$. As the virtual frames are shown, the drop D1' moves and hangs on the middle spindle-knot. At $\sim 967.0 \mathrm{~ms}$, the drop $\mathrm{D} 1^{\prime}$ is merging with drop $\mathrm{D}\left(2^{\prime}+3^{\prime}\right)$. Drop $\mathrm{D}\left(2^{\prime}+3^{\prime}\right)+1^{\prime}$ is hanging on the biggest spindle-knot at $\sim 968.4 \mathrm{~ms}$. The length of movement $\left(\mathrm{L}_{\mathrm{b}}\right)$ is $\sim 2.46 \mathrm{~mm}$ at the whole coalescence process. Even though the two processes of coalescence have the same result that three water drops coalesce into a bigger one with direction movement at the gradient spindle-knot fiber and the transport distances are no obvious disparity $(\sim 2.80 \mathrm{~mm}$ in normal sequence and $\sim 2.46 \mathrm{~mm}$ in reverse sequence), but the reverse sequence spends more longer time $(\sim 968.4 \mathrm{~ms})$ than the normal sequence ( $\sim 26.8 \mathrm{~ms})$. The speed of normal sequence transport $(\sim 0.105 \mathrm{~m}$ $\left.\mathrm{s}^{-1}\right)$ is almost 42 times more than that of reverse sequence transport $\left(\sim 0.0025 \mathrm{~m} \mathrm{~s}^{-1}\right)$ on average. It illustrates that the gradient structure existence of spindle-knots on the fiber decides the action of transport and the volumes and position of water drops effect the speed of transport. 

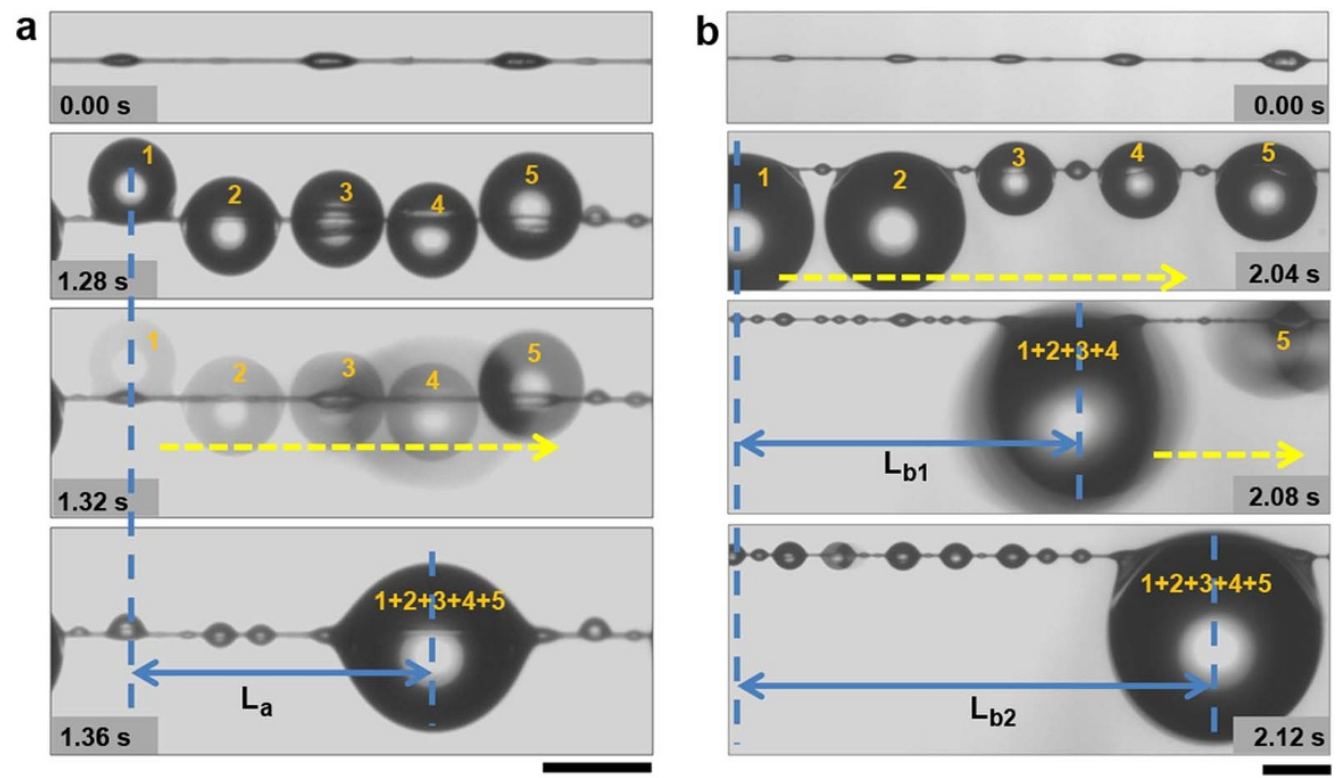

Figure 3 Water drop transport on BFGS with weak/strong gradient spindle-knots. (a), In weak gradient spindle-knots, water drops (1,2,3,4,5) hanging on single spindle-knots and joint, and form a big drop $(1+2+3+4+5)$ via transferring from smaller spindle-knot to bigger ones at $\sim 1.36 \mathrm{~s}$. The transport length $\left(\mathrm{L}_{\mathrm{a}}\right)$ is $\sim 1.41 \mathrm{~mm}$ (see distance between both vertical dash lines) with time of $\sim 1.36 \mathrm{~s}$. (b), In strong gradient spindle-knot, water drops move directionally and hang between two spindle-knots. The length $\left(\mathrm{L}_{\mathrm{b} 1}\right)$ of movement to bigger spindle-knot is $\sim 2.70 \mathrm{~mm}$. The drop is coalesced with small drops $(1,2,3,4)$ at $\sim 2.08 \mathrm{~s}$ and the last drop $(1+2+3+4+5)$ moves to right point location of two biggest spindle-knots (at $\sim 2.12 \mathrm{~s}$ ). The transport length $\left(\mathrm{L}_{\mathrm{b} 2}\right)$ is $\sim 3.76 \mathrm{~mm}$ (see distance between both vertical dash lines) with time of $\sim 2.12 \mathrm{~s}$. Scale bars, $500 \mu \mathrm{m}$.

Velocities of micro-drop transport via coalescence. To clarify the effect of spindle-knot size on water transport ability, the gradient spindle-knot fiber is placed in mist with a fog flow at rate of $\sim 50$ $\mathrm{cm} \mathrm{s}^{-1}$, drop behaviors are recorded by a high speed CCD. Velocities (V) of water drop movement are estimated via two different statistics, i.e., one that drops coalesce on single spindle-knot, which the velocity is defined as $\mathrm{V}_{1}$; other that drops move from small spindle-knot to big one, which the velocity is defined as $\mathrm{V}_{2}$. We choose 55 different size spindle-knots (i.e., the height $(\mathrm{H})$ of spindle-knot ranges from $\sim 28.0$ to $\sim 217.5 \mu \mathrm{m}$, the half angle of that ranges from $\sim 14.7^{\circ}$ to $\sim 30.8^{\circ}$ ) to test the $V_{1}$ of drops coalesce on single spindle-knot at the moment of droplet coalescence (as shown in Figure 5a). Figure 5a shows the statistical relationship between $\mathrm{V}_{1}$ and $\mathrm{H}$ on single spindle-knot, there is a fitting relationship (see Supplementary information, Tab. S3): $\mathrm{V}_{1} \sim[-0.15 \exp (-\mathrm{H} / 204.65)+0.16](\mathrm{m}$ $\mathrm{s}^{-1}$ ), where the $V_{1}$ is increasing with the $H$, estimated $V_{1, \max } \sim 0.1 \mathrm{~m}$ $\mathrm{s}^{-1}$ at $\mathrm{H} \sim 217.5 \mu \mathrm{m}$. A more height can offer a more difference in Laplace pressure, which would drive water drops coalescence with a high velocity. The inset (in Fig. 5a) shows the optical images of droplet movement on one spindle-knot. To reveal further the gradient effect of two spindle-knots on velocity, we select all the pitch of gradient spindle-knots are $\sim 621 \pm 48 \mu \mathrm{m}$, among which the small spindle-knots are chose with the same height of $\sim 56 \pm$ $8 \mu \mathrm{m}\left(\mathrm{H}_{1}\right)$ and the height of big ones $\left(\mathrm{H}_{2}\right)$ range from $\sim 68 \mu \mathrm{m}$ to $\sim 155 \mu \mathrm{m} . \mathrm{H}_{2} / \mathrm{H}_{1}$ can be reflected the extent of gradient between adjoining spindle-knots. Figure $5 \mathrm{~b}$ shows the relationship between $\mathrm{V}_{2}$ and $\mathrm{H}_{2} / \mathrm{H}_{1}$. The inset in Fig. 5b shows the optical images that water drops move from the small spindle-knot to the big one via coalescence. From curve in Fig. $5 b$, the $\mathrm{V}_{2}$ is rapidly increasing with $\mathrm{H}_{2} / \mathrm{H}_{1}$, and until $\mathrm{H}_{2} / \mathrm{H}_{1}$ reaches 2.25 , subsequently, slows down. It is estimated $\mathrm{V}_{2, \max } \sim 0.22 \mathrm{~m} \mathrm{~s}^{-1}$ at $\mathrm{H}_{2} / \mathrm{H}_{1} \sim 2.25$. This indicates that the difference of Laplace pressure plays an important role in driving water drops movement on gradient spindle-knots to extent.

\section{Discussion}

To further reveal how the water drops move directionally on the BFGS via two modes above, we select a gradient spindle-knot fiber with two spindle-knots, which the size of two spindle-knots (Fig. 6a, the top) are respectively of height of $\sim 178 \mu \mathrm{m}$ (S1), $\sim 249 \mu \mathrm{m}$ (S2) and width of $\sim 498 \mu \mathrm{m}$ (S1), $\sim 747 \mu \mathrm{m}$ (S2). The largest pitch is $\sim 1.1 \mathrm{~mm}$. Figure $6 \mathrm{a}$ shows the movement processes of two water drops hanging on two spindle-knots, respectively. Two drops grow on two spindle-knots, respectively (Fig. 6a, at $0 \mathrm{~ms}$ ), until they are big enough to coalesce into one bigger one (Fig. $6 \mathrm{a}$, at $\sim 1.4 \mathrm{~ms}$ ). As two water drops will release energy in the coalescence process, the bigger water drop is in an unstable state which results in a large deformation to form the most stable state. It is reported a continuous diametergradient cone fiber makes the drop move toward direction of larger diameter ${ }^{32}$ and rough surface of fiber generates the capillary adhesion to regulate the behavior of hanging-drop ${ }^{3}$. Here, the diameter of S2 is more than S1, for S2 can provide a more capillary adhesion. The water droplet hanging on S2 is more stable than on S1 (Fig. 6a, at $\sim 4.2-12.6 \mathrm{~ms}$ ). At last, the water is hanging on the S2 stably (Fig. $6 \mathrm{a}$, at $\sim 23.8 \mathrm{~ms}$ ). This process makes the water drop look for a most stable state and move from the small size spindle-knot to the bigger one (see the blue lines in Fig. 6a). The length of movement $\left(\mathrm{L}_{\mathrm{a}}\right)$ is $\sim 1.86 \mathrm{~mm}$ with time of $\sim 23.8 \mathrm{~ms}$. With the growth of these drops and others around it, it will duplicate this process to coalesce into bigger one and move toward bigger spindle-knots. In this process, the coalescence offers the energy to move, and the gradient spindle-knots further promote the movement by gradient of capillary adhesion and meantime play a role in stabilizing the water drops. The dynamic process of the coalesced drop transport can be seen in Supplementary Movie S1 (the true time of the process is $\sim 0.048 \mathrm{~s}$ ). Figure $6 \mathrm{~b}$ shows optical images of the dynamic process that how a water drop hanging between two different size spindle-knots moves towards bigger spindle-knot via in-situ observation by CCD. Two spindle-knots are respectively defined S1 and S2 (Fig. 6b, the top, the same with Fig. $6 a$, the top). Here, $\alpha 1$ is the angle of liquid surface and S1. And $\alpha 2$ is the angle of liquid surface and S2. At $0 \mathrm{~s}$, the water drop is hanging stably between two spindle-knots with different sizes. The $\alpha 1$ $\left(\sim 110^{\circ}\right)$ is more than the $\alpha 2\left(\sim 66^{\circ}\right)$ because the size of S1 is less than that of S2 (Fig. 6b, top). Carroll ${ }^{33-35}$ and Mchale et $\mathrm{al}^{36}$. describe that the gravity $(G)$ can be balanced with capillary forces $\left(f_{c}\right)$ when 
a

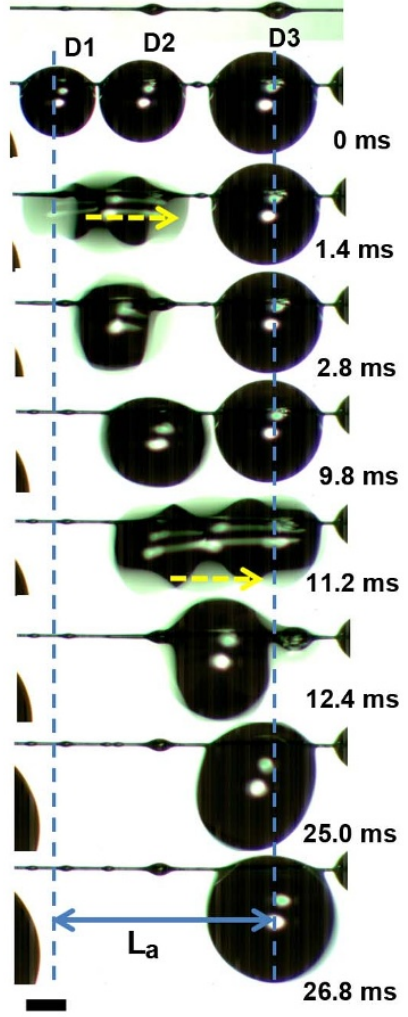

b

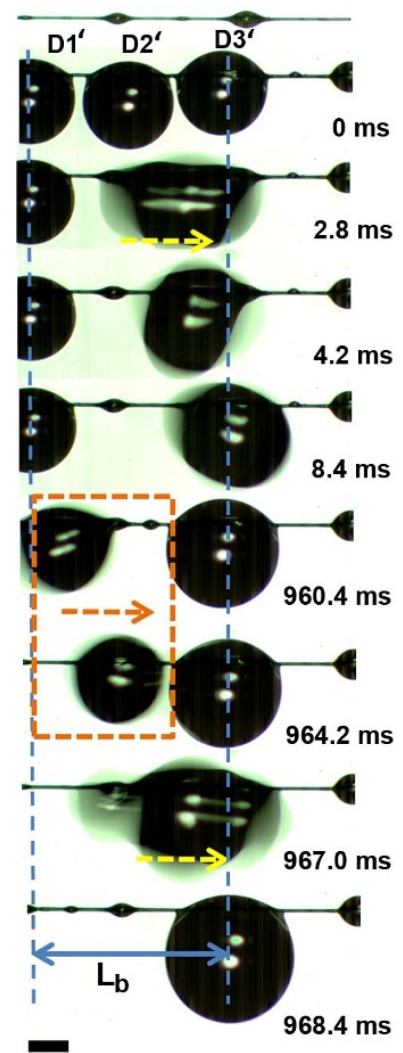

Figure $4 \mid$ Water direction tendency coalescences in normal sequence and reverse sequence. (a), In normal sequence water drops coalescence with the process of $(\mathrm{D} 1+\mathrm{D} 2)+\mathrm{D} 3$. The drop D1 coalesces with drop D2 to form Drop $\mathrm{D}(1+2)$, and it moves towards bigger spindle-knots. With the water drops growing, drop $\mathrm{D}(1+2)$ is merging with drop $\mathrm{D} 3$ to form drop $\mathrm{D}(1+2)+3$. The length of movement $\left(\mathrm{L}_{\mathrm{a}}\right)$ is $\sim 2.80 \mathrm{~mm}$ with $\sim 26.8 \mathrm{~ms}$. (b), The reverse sequence of water drops coalescence. The drop D2' and D3' firstly combine together form the drop $\mathrm{D}\left(2^{\prime}+3^{\prime}\right)$. With the drops growing, the drop D1' moves towards to the right due to the first mode (virtual frame) and coalesces with drop $\mathrm{D}\left(2^{\prime}+3^{\prime}\right)$ to form Drop D $\left(2^{\prime}+\right.$ $\left.3^{\prime}\right)+1^{\prime}$. The length of movement $\left(\mathrm{L}_{\mathrm{b}}\right)$ is $\sim 2.46 \mathrm{~mm}$ with $\sim 968.4 \mathrm{~ms}$. Scale bars, $500 \mu \mathrm{m}$.

water droplets are hung on the fibers. On the one hand, the capillary forces are proportional to the curvature of the liquid surface. The more is curvature of the liquid surface, the more are capillary forces; or vice versa. The angle $\alpha 2\left(\sim 70^{\circ}\right)$ is less than $\alpha 1\left(\sim 91^{\circ}\right.$, Fig. $6 \mathrm{~b}$, at $\sim 4.2 \mathrm{~ms}$ ), and the curvature of liquid surface with $\mathrm{S} 1$ is more than that of S2. So the water drop at S1 site is moving towards to right. The angle $\alpha 1$ is decreasing in the process for a stable state. At S1 site, due to Laplace pressure gradient, the water drop easily moves from joint site to the hump (Fig. $6 \mathrm{~b}$, at $\sim 4.2 \mathrm{~ms}$ ). With the angle $\alpha 1$ decreasing, the Laplace pressure at the contact line tends to increase. It is quite difficult for the contact line to pass through location of hump. But the capillary force gradient (at S1 and S2) overcomes Laplace pressure gradient. The water drop moves from hump to the right joint site. The angle $\alpha 1\left(\sim 84^{\circ}\right)$ is decreasing and $\alpha 2\left(\sim 69^{\circ}\right.$, Fig. $6 \mathrm{~b}$, at $\left.\sim 5.6 \mathrm{~ms}\right)$ is essentially unchanged. On the other hand, a larger pearly hangingdrop is regulated by a spindle-knot due to the roughness and curvature (Fig. $1 b$ ). The roughness can enhance the pinning effect to three-phase contact line and thus cooperate with the effects of curvature and slope of hump to hang a larger volume of the extreme drop stably. However, when the liquid surface leaves S1 site (smaller spindle-knot), there is no recession barrier of contact line hanging on a uniform location. It results in an instable three-phase contact line which leads it easily sliding from spindle-knot site to the uniform location (Fig. 6b, at $\sim 7 \mathrm{~ms}$ ). The capillary force at this location

decreases rapidly, and the capillary force at S2 site is unchanged $\left(\alpha 1 \sim 46^{\circ}, \alpha 2 \sim 63^{\circ}\right)$. In this process, the water drop moves toward to bigger spindle-knot (Fig. $6 \mathrm{~b}$, at $0 \mathrm{~ms}, \alpha 1 \sim 38^{\circ}, \alpha 2 \sim 70^{\circ}$ ). At $\sim 21.0 \mathrm{~ms}$, the water droplet is hanging on the bigger spindle-knot stably. The angle $\alpha 1$ and $\alpha 2$ are $\sim 47^{\circ}$ and $\sim 55^{\circ}$, respectively, which is little difference. Tian et $\mathrm{al}^{37}$. demonstrated that uniform spindleknots fiber can stably pin a water drop through the combination of "slope" and "curvature" effects. The gradient spindle-knots structure successfully breaks the stability offered by two uniform spindleknots, which leads to move. The length of movement $\left(\mathrm{L}_{b}\right)$ is $\sim 0.873 \mathrm{~mm}$ with time of $\sim 21.0 \mathrm{~ms}$. Cooperation of two modes, the water drop can move on the gradient fiber from smaller spindle-knot to bigger one. There are thereby three factors to drive the water drop movement along the gradient fiber: i.e., capillary adhesion force $\left(F_{\mathrm{ca}}\right)$, difference in Laplace pressure $\left(F_{\mathrm{lp}}\right)$ along the gradient spindle-knots and also drops coalescence release energy $\left(F_{\mathrm{cr}}\right)$ as well. The capillary force that is induced by the roughness according Wenzel's law ${ }^{38}$ is described as follows: $F_{\mathrm{ca}}=r \cdot 4 \pi \mathrm{b} \gamma$ $\sin (\pi-\alpha)$, where $F_{\mathrm{ca}}$ is the capillary force of spindle-knots; $r$ is the roughness; the $b$ is the effective radius of silk $\left(b_{(\text {small knot) }}<b_{(\text {big knot })}\right)$ and $\alpha$ is the angle of liquid surface and spindle-knot. When $\alpha$ is $\sim 90^{\circ}$, the $\sin (\pi-\alpha)$ reaches the max value. The $\alpha 1$ decreases from $\sim 110^{\circ}$ to $\sim 91^{\circ}$ (meantime move from left joint to the hump site) which leads to the capillary adhesion force increasing to prevent the drop movement from left to right. Until the liquid surface passes the hump to right joints (the $b$ decreases sharply), the capillary adhesion force of smaller spindle-knot is less than that of bigger one. The capillary adhesion force difference drives the water drops movement towards to bigger spindle-knots. The second driving force is difference in Laplace pressure $\left(F_{\mathrm{lp}}\right)$ described as $F_{\mathrm{lp}}=-\int \frac{2 \gamma}{\left(r+R_{0}\right)^{2}} \sin \beta d z$, where $\gamma$ is the surface tension of water; $r$ is the local radius; $R_{0}$ is the drop radius; $\beta$ is the half apex-angle of the spindle-knot $\left(\beta_{\text {(small knot) }}<\right.$

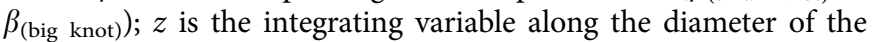
spindle-knot. The Laplace pressure on the high curvature site is larger than that on the low curvature site. Firstly, the $F_{\text {lp }}$ promotes tiny water drops moving from uniform fiber to the joint and then to the spindle-knot because the radius of uniform fiber $\left(r_{u}\right)$ is smaller than that of joint $\left(r_{j}\right)$; the $r_{j}$ is less than the radius of spindle-knot $\left(r_{s}\right)$. Secondly, $F_{l p}$ also contributes to propelling the drop transport from small spindle-knot to big one due to $F_{\mathrm{lp} \text { (small spindle-knot) }}<F_{\mathrm{lp} \text { (big }}$ spindle-knot). The third force is drops coalescence release energy, which assists the previous forces due to the gradient structure on the fiber. Cooperation of three forces acts on these two modes to drive water drops directional movement. Figure 7 illustrates the mechanism of the water drops transport along the BFGS in a long distance. Fig. 7a-b indicates the case of the directional driving of tiny water condensed drops toward spindle-knot by the cooperation of surface energy in gradient and Laplace pressure in difference, similar to the wetted spider silk. Fig. 7c shows the first mode of water drops transport. The cooperation of difference in Laplace pressure $\left(F_{\mathrm{lp}}\right)$ along the gradient spindle-knots and drops coalescence release energy $\left(F_{\mathrm{cr}}\right)$ drives the coalescence water transporting towards big spindle-knot for a stable condition (Fig. 7c). When a bigger water drop hangs on one spindle-knot, it tends to coalesce into another water drop on the biggest spindle-knot. A coalescence water drop hanging between two spindle-knots (Fig. 7d) tends to slide from the joint of smaller spindle-knot towards the biggest one because of $F_{\text {ca(small spindle-knot) }}$ $<F_{\text {ca(big spindle-knot), }}$, resulting in the angle of liquid surface and small spindle-knot $\alpha 1$ reducing. The second mode prompts the bigger water drop transporting towards the biggest spindle-knot and hanging on it (Fig. 7e). Cooperation of both modes drive the water drops move directionally on the BFGS in a long distance.

BFGSs display an excellent fast micro-drop transport in a long distance due to multi-level cooperation of capillary adhesion force, difference in Laplace pressure and drops coalescence release energy. 

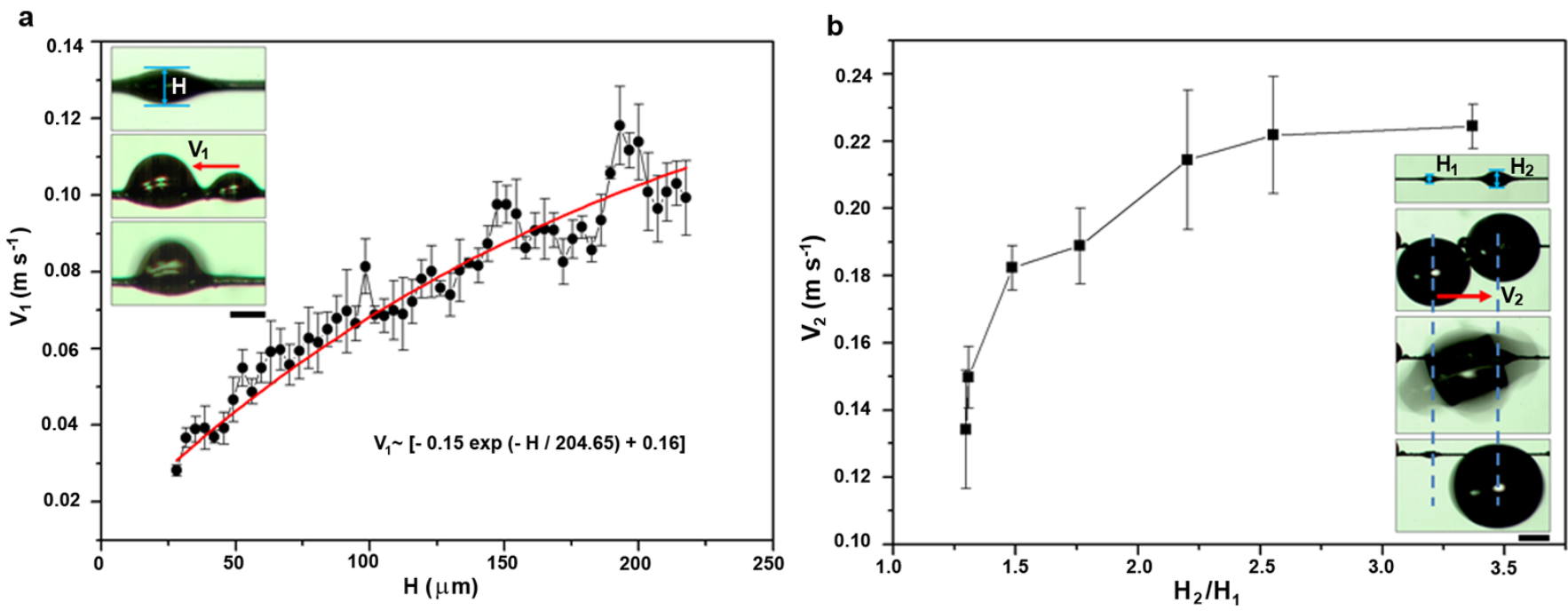

Figure 5 Velocities of water drop coalescence on one spindle-knot and two adjacent spindle-knots. (a), Relationship of velocity $\left(\mathrm{V}_{1}\right)$ and height $(\mathrm{H})$ on one spindle-knot. The $\mathrm{V}_{1}$ is increasing with $\mathrm{H}$, there is a fitting relationship: $\mathrm{V}_{1} \sim[-0.15 \exp (-\mathrm{H} / 204.65)+0.16]\left(\mathrm{m} \mathrm{s}^{-1}\right)$. It is estimated $\mathrm{V}_{1}$, max $\sim 0.1 \mathrm{~m}$ $\mathrm{s}^{-1}$ at $\mathrm{H} \sim 217.5 \mu \mathrm{m}$. (b), Relationship of velocity $\left(\mathrm{V}_{2}\right)$ and height ratio $\left(\mathrm{H}_{2} / \mathrm{H}_{1}\right)$ of adjacent spindle-knots. The $\mathrm{V}_{2}$ is rapidly increasing with the $\mathrm{H}_{2} / \mathrm{H}_{1}$, until $\mathrm{H}_{2} / \mathrm{H}_{1} \sim 2.25$, subsequently, slows down. It is estimated $\mathrm{V}_{2 \text {, max }} \sim 0.22 \mathrm{~m} \mathrm{~s}^{-1}$ at $\mathrm{H}_{2} / \mathrm{H}_{1} \sim 2.25$. The insets are the optical images of drop movement process. Scale bar, a, $150 \mu \mathrm{m}, \mathrm{b}, 500 \mu \mathrm{m}$.

The investigation of relationship between gradient structures and water transport may help us to design novel materials for a potential applications such as the fluid controlling in water-harvesting projects $^{39-41}$ and filtering aerosols systems ${ }^{42}$.

\section{Methods}

The bioinspired fibers with gradient spindle-knots (BFGS) can be fabricated by the tilted dip-coating method combining Rayleigh instability technique ${ }^{1,2}$. The condition
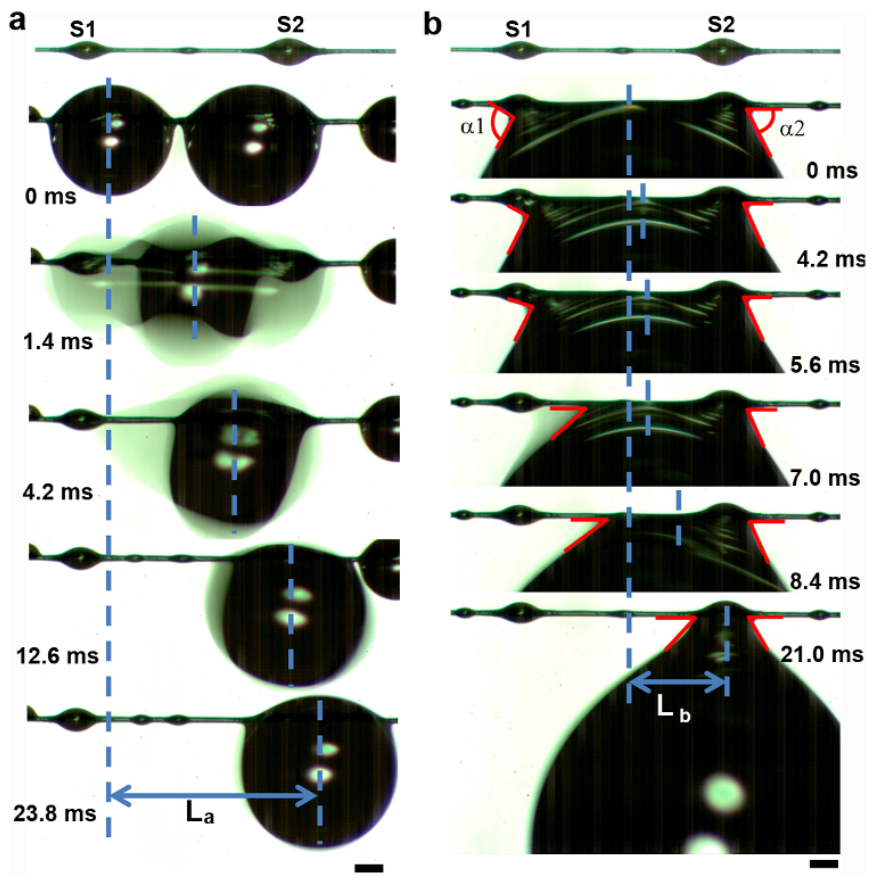

Figure 6 | Dynamic behaviors on both modes of water drop movement in direction. (a), The movement process of water drops hanging on a single spindle-knot. The length of movement $\left(\mathrm{L}_{\mathrm{a}}\right)$ is $\sim 1.86 \mathrm{~mm}$ with time of $\sim 23.8 \mathrm{~ms}$. (b), Water drops directional movement between two spindleknots with different sizes. $\alpha 1$ and $\alpha 2$ are the angle of liquid surface with S1 and $S 2$ respectively. The angle $\alpha 1$ is decreasing in the process for a stable state and $\alpha 2$ is almost unchanged. The length of movement $\left(\mathrm{L}_{\mathrm{b}}\right)$ is $\sim 0.873 \mathrm{~mm}$ with time of $\sim 21.0 \mathrm{~ms}$. Scale bars, $250 \mu \mathrm{m}$. of fabricating gradient spindle-knots fibers can be optimized by tuning the concentrations of PVDF polymer solutions ranged from $7 \%, 8 \%, 9 \%$ to $10 \%$ (weight percentage) and dip-coating tilted angles ranged from $0^{\circ}, 5^{\circ}, 10^{\circ}$, to $15^{\circ}$ to horizontal line. The whole process of fabrication is recorded by a using a high-speed CCD camera. After tilted dip-coating and dryness of fiber in ambient environment, BFGS is achieved successfully. The structures of BFGS are observed by scanning electron microscopy (SEM) at $10 \mathrm{kV}$. The tiny water drops are generated on BFGS via an ultrasonic humidifier with a fog flow. The dynamic processes of micro-drop transport

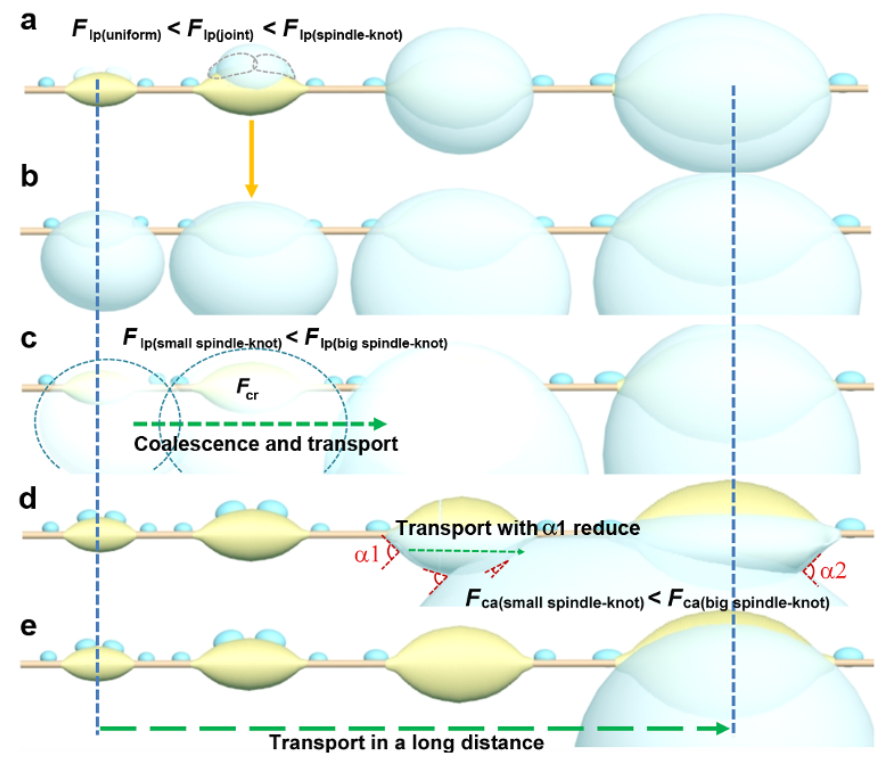

Figure $7 \mid$ Illustration of water drops transport in a long distance. (a-b), The directional driving of tiny water condense drops toward spindle-knot by the cooperation of surface energy in gradient and Laplace pressure in difference. (c), The first mode drives the coalescence water transporting towards big spindle-knot for a stable condition via the cooperation of difference in Laplace pressure $\left(F_{\mathrm{lp}}\right)$ along the gradient spindle-knots and drops coalescence release energy $\left(F_{\mathrm{cr}}\right)$. (d), A coalescence water drop hanging between two spindle-knots tends to slide from the joint of smaller spindle-knot towards the biggest one because of $F_{\text {ca(small spindle-knot) }}<$ $F_{\text {ca(big spindle-knot) }}$ resulting in the angle of liquid surface and small spindleknot $\alpha 1$ reducing. (e), The second mode prompts the bigger water drop transporting towards the biggest spindle-knot and hanging on it. 
are captured by a high-speed CCD camera. Statistical analysis is done by the analyst of origin8 software.

1. Parker, A. R. \& Lawrence, C. R. Water capture by a desert beetle. Nature 414, 33-33 (2001).

2. Zheng,Y. et al. Directional water collection on wetted spider silk. Nature 463, 640-643 (2010).

3. Huang, Z., Chen, Y., Zheng, Y. \& Jiang, L. Capillary adhesion of wetted cribellate spider capture silks for larger pearly hanging-drops. Soft Matter 7, 9468-9473 (2011).

4. Ebner, M., Miranda, T. \& Roth-Nebelsick, A. Efficient fog harvesting by Stipagrostis sabulicola (Namib Desert bushman grass). J. Arid Environ. 75 524-531 (2011).

5. Andrews, H. G., Eccles, E. A., Schofield, W. C. E. \& Badyal, J. P. S. Threedimensional hierarchical structures for fog harvesting. Langmuir 27, 3798-3802 (2011).

6. Ju, J., Bai, H., Zheng, Y., Zhao, T. \& Jiang, L. A multi-structural and multifunctional integrated fog collection system in cactus. Nat. Commun. 3, 1247-1252 (2012).

7. Zhao, Q. \& Buehler, M. J. Spider silk: Webs measure up. Nat. Mater. 12, 185-187 (2013).

8. Lorenceau, É. \& Quéré, D. Drops on a conical wire. J. Fluid Mech. 510, 29-45 (2004).

9. Yao, X., Song, Y. \& Jiang, L. Applications of bio-inspired special wettable surfaces. Adv. Mater. 23, 719-734 (2011).

10. Liu, K., Yao, X. \& Jiang, L. Recent developments in bio-inspired special wettability. Chem. Soc. Rev. 39, 3240-3255 (2010).

11. Wang, J., Zhang, Y., Wang, S., Song, Y. \& Jiang, L. Bio-inspired colloidal photonic crystals with controllable wettability. Accounts Chem. Res. 44, 405-415 (2011).

12. Sun, T., Qing, G., Su, B. \& Jiang, L. Functional biointerface materials inspired from nature. Chem. Soc. Rev. 40, 2909-2921 (2011).

13. Bai, H. et al. Direction controlled driving of tiny water drops on bioinspired artificial spider silks. Adv. Mater. 22, 5521-5525 (2010).

14. Bai, H. et al. Controlled fabrication and water collection ability of bioinspired artificial spider silks. Adv. Mater. 23, 3708-3711 (2011).

15. Tian, X., Bai, H., Zheng, Y. \& Jiang, L. Bio-inspired feterostructured bead-onstring fibers that respond to environmental wetting. Adv. Funct. Mater. 21, 1398-1420 (2011)

16. Chaudhury, M. K. \& Whitesides, G. M. How to make water run uphill. Science 256 1539-1541 (1992).

17. Daniel, S., Chaudhury, M. K. \& Chen, J. C. Fast drop movements resulting from the phase change on a gradient surface. Science 291, 633-636 (2001).

18. Daniel, S., Sircar, S., Gliem, J. \& Chaudhury, M. K. Ratcheting motion of liquid drops on gradient surfaces. Langmuir 20, 4085-4092 (2004).

19. Bai, H. et al. Large-scale fabrication of bioinspired fibers for directional water collection. Small 7, 3429-3433 (2011).

20. Kang, E., Jeong, G. S., Choi, Y. Y., Lee, K. H. \& Lee, S. Digitally tunable physicochemical coding of material composition and topography in continuous microfibers. Nat. Mater. 10, 877-883 (2011).

21. Chen, Y., Wang, L., Xue, Y., Zheng, Y. \& Jiang, L. Bioinspired spindle-knotted fibers with a strong water-collecting ability from a humid environment. Soft Matter 8, 11450-11454 (2012).

22. Yang, H. et al. Temperature-triggered collection and release of water from fogs by a sponge-like cotton fabric. Adv. Mater. 25, 1150-1154 (2013).

23. Tian, E., Wang, J., Zheng, Y., Song, Y. \& Jiang, L. Colorful humidity-sensitive photonic crystal hydrogel. J. Mater. Chem. 18, 1116-1122 (2008).

24. Guo, L., Jiang, H. \& Sun, H. Two-beam-laser interference mediated reduction, patterning and nanostructuring of graphene oxide for the production of a flexible humidity sensing device. Carbon 50, 1667-1673 (2012).

25. Bai, H., Ju, J., Zheng, Y. \& Jiang, L. Functional fibers with unique wettability inspired by spider silks. Adv. Mater. 24, 2786-2791 (2012).
26. Dubois, P. et al. Ionic liquid droplet as e-microreactor. Anal. Chem. 78, 4909-4917 (2006).

27. Rayleigh, L. On the instability of jets. Proc. London Math. Soc. 10, 4-13 (1878).

28. Carroll, B. J. \& Lucassen, J. Effect of surface dynamics on the process of droplet formation from supported and free liquid cylinders. J. Chem. Soc. Faraday Trans. 70, 1228-1239 (1974)

29. de Ryck, A. \& Quéré, D. Fluid coating from a polymer solution. Langmuir 14, 1911-1914 (1998).

30. Hou, Y. et al. Stronger water hanging ability and higher water collection efficiency of bioinspired fiber with multi-gradient and multi-scale spindle knots. Soft Matter 8, 11236-11239 (2012)

31. Hou, Y., Chen, Y., Xue, Y., Zheng, Y. \& Jiang, L. Water collection behavior and hanging ability of bioinspired fiber. Langmuir 28, 4737-4743 (2012).

32. Lorenceau, E., Clanet, C. \& Quéré, D. Capturing drops with a thin fiber. J. Colloid Int. Sci. 279, 192-197 (2004).

33. Carroll, B. J. The accurate measurement of contact angle, phase contact areas, drop volume, and Laplace excess pressure in drop-on-fiber. J. Colloid Int. Sci. 57, 488-495 (1976).

34. Carroll, B. J. The equilibrium of liquid drops on smooth and rough circular cylinders. J. Colloid Int. Sci. 97, 195-200 (1984).

35. Carroll, B. J. Equilibrium conformations of liquid drops on thin cylinders under forces of capillarity. A theory for the roll-up process. Langmuir 2, 248-250 (1986).

36. McHale, G., Kab, N. A., Newton, M. I. \& Rowan, S. M. Wetting of a high-energy fiber surface. I. Colloid Int. Sci. 186, 453-461 (1997).

37. Tian, X., Chen, Y., Zheng, Y., Bai, H. \& Jiang, L. Controlling water capture of bioinspired fibers with hump structures. Adv. Mater. 23, 5486-5491 (2011).

38. Wenzel, R. N. Resistance of solid surfaces to wetting by water. Ind. Eng. Chem. $\mathbf{2 8}$ 988-994 (1936).

39. Estrela, M., Valiente, J., Corell, D. \& Millán, M. M. Fog collection in the western Mediterranean basin (Valencia region, Spain). Atmos. Res. 87, 324-337 (2008).

40. Molina, J. M. \& Escobar, C. M. Fog collection variability in the Andean mountain range of Southern Colombia. Special Issue: Fog Research 139, 127-140 (2008).

41. Vince, G. Out of the mist. Science 330, 750-751 (2011).

42. Agranovski, I. E., Moustafa, S. \& Braddock, R. D. Performance of activeted carbon loaded fibrous filters on simultaneous removal of particulate and gaseous pollutants. Environ. Technol. 26, 757-766 (2005).

\section{Acknowledgements}

This work is supported by National Research Fund for Fundamental Key Project (2013CB933000, 2010CB934700), National Natural Science Foundation of China (21234001), Doctoral Fund of Ministry of Education of China (20121102110035), and Graduate Student Innovation Practice Foundation of Beihang University.

\section{Author contributions}

Y.Z. conceived the research, Y.C. and L.W. conducted the experiments, Y.X. and L.J. analysed data. Y.Z. and Y.C. wrote the paper.

\section{Additional information}

Supplementary information accompanies this paper at http://www.nature.com/ scientificreports

Competing financial interests: The authors declare no competing financial interests.

How to cite this article: Chen, Y., Wang, L., Xue, Y., Jiang, L. \& Zheng, Y. Bioinspired tilt-angle fabricated structure gradient fibers: micro-drops fast transport in a long-distance. Sci. Rep. 3, 2927; DOI:10.1038/srep02927 (2013)

\footnotetext{
cc)(1) (2) This work is licensed under a Creative Commons Attribution-

cc. visit http://creativecommons.org/licenses/by-nc-sa/3.0
} 